\title{
Recombinant PRAME Protein Plus AS15 Adjuvant GSK2302025A
}

National Cancer Institute

\section{Source}

National Cancer Institute. Recombinant PRAME Protein Plus AS15 Adjuvant

GSK2302025A. NCI Thesaurus. Code C92586.

A recombinant form of the human PRAME (Preferentially Expressed Antigen of

Melanoma) protein combined with the AS15 adjuvant, with potential immunostimulatory and antineoplastic activities. Upon intramuscular administration, GSK2302025A may stimulate the host immune response to mount a cytotoxic T-lymphocyte $(C T L)$ response against tumor cells that overexpress the PRAME protein, resulting in tumor cell lysis. The tumor-associated antigen PRAME is often overexpressed by a variety of tumor cell types. AS15 is an potent adjuvant liposomal formulation that contains CpG 7909, monophosphoryl lipid, and QS-21. 